\title{
Atração miraxonal exercida por Biomphalaria tenagophila selecionada geneticamente para o caráter susceptibilidade ${ }^{*}$

\author{
Miraxonal atraction exercised by Biomphalaria tenagophila genetically selected \\ for susceptibility
}

\author{
Eliana Maria Zanotti-Magalhães**, Luiz Augusto Magaihães**, José Ferreira de Carvalho***
}

\begin{abstract}
ZANOTTI-MAGALHÃES, E. M. et al. Atração miraxonal exercida por Biomphalaria tenagophila selecionada geneticamente para o caráter susceptibilidade. Rev. Saúde públ., S. Paulo, 25: 134-8, 1991. Tratou-se de testar a atração miraxonal excrcida nas gerações de $B$. tenagophila, selecionadas ou não selecionadas, para o caráter susceptibilidade à infecção por S. mansoni SJ, sobre larvas do trematódeo da mesma linhagem, obtidas de moluscos selecionados e não selecionados para o mesmo caráter. Foi utilizado um aparelho de vidro, já testado anteriormente, constituído de duas câmaras ligadas por um canal e os caramujos ou sua água de condicionamento ( $\mathrm{SCW}$ ), colocados aleatoriamente em uma das câmaras. Observou-se em lupa estercoscópica a movimentação dos miracídios por $15 \mathrm{~min}$. A análise dos resulıados mostrou que a geração do molusco (selecionado ou não selecionado) foi o único fator importante na atração: os moluscos sclecionados para o caráter susceptibilidade ou sua SCW, atraíram mais os miracídios.
\end{abstract}

Descritores: Biomphalaria, fisiologia. Schistosoma mansoni, fisiologia. Alividade molora.

\section{Introduçăo}

Desde 1958, sabc-se, através da observação de Kloetzel7, que miracídios de Schistosoma mansoni apresentam quimiotropismo para moluscos $B$. glabrata. Posteriormentc, Chernin 2,4 (1970, 1974) estudou a ação de substâncias emanadas de $B$. glabrata sobre os miracídios, não evidenciando porém a fonte e a estrutura das substâncias atracntcs. Brasio e col. ${ }^{1}$ (1985) demonstraram que a auração exercida por $B$. glabrata foi mais intensa do que a exercida por $B$. tenagophila quando estes moluscos foram colocados frente a miracídios de populaçōes simpátricas. Verifica-se que $B$. glabrata apresenta, geralmente uma maior susceptibilidade à infecção do que $B$. tenagophila. O estudo destes aspectos adquiriu maior importância epidemiológica com o

* Publicação financiada pela FAPESP

** Departamento de Parasitologia do Instituto de Biologia da Universidade Estadual de Campisas (UNICAMP)- Campinas, SP - Brasil.

*** Departamento de Estatística do Instituto de Matemática, Estatística e Ciência da Computação da UNICAMP Campinas, SP - Brasil.

Separadas/Reprints: E. M. Zanoui-Magalhães - Caixa Postal 6109, Cidade Universitária, Barão Geraldo - 13081 - Campinas, SP - Brasil. trabalho de Zanoti-Magalhães (1987), no qual se verificou uma correlação entre a susceptibilidade do velor e o grau de patogenicidade do trematódeo no hospedeiro vertcbrado. Contudo, não se conhece a relação entre o grau de susceptibilidade apresentado pelo molusco à infecção por $S$. manso$n i$ e a intensidade da atração miraxonal. No presente trabalho, utilizou-se populações de $B$. tenagophila selecionadas gencticamente para o caráter susceptibilidade, pretendendo verificar se a maior susceptibilidade do molusco correspondia a um maior poder de atração sobre os miracidios.

\section{Material e Método}

Foram utilizados moluscos B. tenagophila criados no moluscário do Departamento de Parasitologia da UNICAMP, descendentes de caramujos provenientes do Vale do Rio Paraíba do Sul, Estado de São Paulo. A fim de se obter moluscos geneticamente selecionados, foi utilizada a autofecundação de moluscos susceptívcis obıendo-se progenies que compuseram populaçōes que apresentavam altas taxas de susceptibilidade (ZanotiMagalhãcs ${ }^{8}$, 1987). Os moluscos sclccionados para o carátcr susceptibilidade utilizados no experimento pertenciam a $16^{\mathrm{a}}$ gcração. Foram utilizados também moluscos da geração parcntal, não 
selecionados, provenientes de fecundação cruzada.

Foram utilizadas duas populações de miracídios de S. mansoni da linhagem SJ, simpátrica dos moluscos usados no experimento. A população parental de miracídios foi proveniente da linhagem rotineiramente mantida no Laboratório de $\mathrm{Pa}$ rasitologia da UNICAMP. A população selccionada de miracídios foi da $16^{a}$ geração de esquistossomos progressivamente mantidos $\mathrm{em}$ moluscos selecionados por autofecundação para 0 caráter susceptibilidade (Zanotti-Magalhães ${ }^{8}$, 1987).

- Além dos moluscos e miracídios, foi utilizada também água de condicionamento (SCW - "snail conditioned water"), obtida de um "pool" de moluscos (Chernin $\left.{ }^{3}, 1972\right)$, das duas populações de moluscos.

Os experimentos foram realizados utilizando-sc um artefato de vidro composto de duas câmaras circulares $(A$ e B) com $30 \mathrm{~mm}$ de diâmetro e 20 $\mathrm{mm}$ de profundidade, unidas por um canal (C) de $40 \mathrm{~mm}$ de comprimento, $11 \mathrm{~mm}$ de largura e 10 $\mathrm{mm}$ de profundidade (Brasio c col'., 1985).

Nas câmaras foram colocados os moluscos ou suas SCW e no canal os miracídios.

Foram rcalizados ensaios testemunhos, utilizando-se somente água declorada nas câmaras A e B.

Após a deposição de 10 miracídios no canal $\mathrm{c}$ a colocação de moluscos ou SCW alcatoriamente em uma das câmaras, foi observado, cm lupa estereoscópica munida com lente frontal $(X 0,5), 0$ comportamento dos miracídios por 15 minutos. Os ensaios foram repetidos dez vezes, utilizando-se novos miracídios, moluscos ou SCW. Os cxperimentos foram realizados com iluminação abundante, homogcamente dispersa.

\section{Resultados}

A taxa de infecção dos moluscos sclecionados foi muito maior do que aquela verificada para os moluscos da geração parcntal (Tabcla 1).

Todos os moluscos, quer sclecionados ou não, atraíram miracídios da geração parental e da

Tabela 1. Taxa de infecção apresentada por $B$. tenagophila das geraçóes parental e $F_{16}$ expostas aos miracidios de $S$. mansoni das geraçōes parental e $F_{16}$.

\begin{tabular}{lllc}
\hline Geração & Moluscos & Moluscos & Taxa de \\
do molusco & infectados & positivos & infecção (\%) \\
\hline
\end{tabular}

\begin{tabular}{llll} 
Parental & 25 & 6 & 24,0 \\
$F_{16}$ & 27 & 26 & 96,3 \\
\hline
\end{tabular}

Tabela 2. Atração miraxonal exercida por moluscos B. tenagophila das geraçōes parental e $F_{16}$, colocados na cámara $A$, frente a miracidios de $S$. mansoni da geraçáo parental.

\begin{tabular}{lccc}
\hline \multirow{2}{*}{$\begin{array}{l}\text { Geração } \\
\text { dos moluscos }\end{array}$} & \multicolumn{3}{c}{ Número de miracidios* } \\
\cline { 2 - 4 } & Câmara A & Câmara B & Canal C \\
\hline Parental & 7 & 3 & 0 \\
& 8 & 1 & 1 \\
& 6 & 3 & 1 \\
& 7 & 3 & 0 \\
& 7 & 2 & 1 \\
& 7 & 2 & 1 \\
& 7 & 2 & 1 \\
Média & 7 & 3 & 0 \\
\hline F16 & 8 & 2 & 0 \\
& 7 & 3 & 0 \\
& 7,1 & 2,4 & 0,5 \\
\hline & 9 & 1 & 1 \\
& 8 & 0 & 1 \\
& 8 & 0 & 2 \\
& 9 & 0 & 1 \\
& 8 & 2 & 0 \\
& 8 & 1 & 1 \\
& 7 & 1 & 2 \\
Média & 8 & 0 & 2 \\
\hline & 8 & 0 & 2 \\
\hline
\end{tabular}

- Número de miracídios observados em cada uma das câmaras após $15 \mathrm{~min}$ de deposição de 10 larvas no canal $\mathrm{C}$.

Tabela 3 . Atração miraxonal exercida por moluscos $B$. tenagophila das geraçōes parental e $F_{16}$, colocados na câmara A, frente a miracídios de S. mansoni da geração $F_{16}$.

\begin{tabular}{|c|c|c|c|}
\hline \multirow{2}{*}{$\begin{array}{l}\text { Geração } \\
\text { dos moluscos }\end{array}$} & \multicolumn{3}{|c|}{ Número de miracidios" } \\
\hline & Câmara A & Câmara B & Canal C \\
\hline Parental & $\begin{array}{l}6 \\
6 \\
7 \\
7 \\
7 \\
8 \\
7 \\
7 \\
6 \\
7\end{array}$ & $\begin{array}{l}3 \\
3 \\
2 \\
3 \\
3 \\
1 \\
3 \\
3 \\
3 \\
3\end{array}$ & $\begin{array}{l}1 \\
1 \\
1 \\
0 \\
0 \\
1 \\
0 \\
0 \\
1 \\
0\end{array}$ \\
\hline Média & 6,8 & 2,7 & 0,5 \\
\hline$F_{16}$ & $\begin{array}{c}10 \\
9 \\
8 \\
9 \\
10 \\
10 \\
9 \\
9 \\
8 \\
9\end{array}$ & $\begin{array}{l}0 \\
1 \\
2 \\
1 \\
0 \\
0 \\
0 \\
0 \\
2 \\
1\end{array}$ & $\begin{array}{l}0 \\
0 \\
0 \\
0 \\
0 \\
0 \\
1 \\
1 \\
0 \\
0\end{array}$ \\
\hline Média & 9,1 & 0,7 & 0,2 \\
\hline
\end{tabular}

Número de miracidios observados em cada uma das câmaras após $15 \mathrm{~min}$ de deposiçāo de 10 larvas no canal $\mathrm{C}$. 
Tabela 4. Atraçáo miraxonal exercida pela água de condicionamento (SCW) de Biomphalaria tenagophila das geraçōes parental e $F_{16}$ colocados na cámara $A$, frente a miracidios de $S$. mansoni da geração parental.

\begin{tabular}{lccc}
\hline Tipo de & \multicolumn{3}{c}{ Número de miracidios** } \\
\cline { 2 - 4 } SCW $^{*}$ & Câmara A & Câmara B & Canal C \\
\hline Parental & 6 & 3 & 1 \\
& 7 & 2 & 1 \\
& 8 & 2 & 0 \\
& 8 & 2 & 0 \\
& 8 & 2 & 0 \\
& 7 & 2 & 1 \\
& 7 & 3 & 0 \\
Média & 7 & 2 & 1 \\
Fis & 7 & 3 & 0 \\
& 8 & 2 & 0 \\
& 7,3 & 2,3 & 0,4 \\
\hline & 7 & 3 & 0 \\
& 7 & 2 & 1 \\
& 8 & 3 & 0 \\
& 10 & 2 & 0 \\
& 9 & 0 & 0 \\
& 8 & 1 & 1 \\
Média & 7 & 3 & 0 \\
& 8 & 2 & 0 \\
& 9 & 1 & 0 \\
\hline
\end{tabular}

" SCW - "snail conditioned water"

* Número de miracidios observados em cada uma das câmaras após 15 min da deposição das larvas no canal $C$.

Tabela 5. Atraçăo miraxonal exercida pela água de condicionamento (SCW) de Biomphalaria tenagophila das geraçoes parental e $F_{16}$ colocados na câmara $A$, frente a miracidios de $S$. mansoni da geraçăo $F_{16}$.

\begin{tabular}{lccc}
\hline Tipo de & \multicolumn{3}{c}{ Número de miracídios ** } \\
\cline { 2 - 4 } SCW* & Câmara A & Câmara B & CanalC \\
\hline Parental & 6 & 3 & 1 \\
& 7 & 2 & 1 \\
& 7 & 2 & 1 \\
& 7 & 3 & 0 \\
& 6 & 3 & 1 \\
& 7 & 3 & 0 \\
& 8 & 2 & 0 \\
Média & 8 & 2 & 0 \\
\hline F. & 7 & 2 & 1 \\
& 7 & 2 & 1 \\
& 7,0 & 2,4 & 0,6 \\
\hline & 10 & 0 & 0 \\
& 10 & 0 & 0 \\
& 9 & 0 & 1 \\
& 8 & 2 & 0 \\
& 10 & 0 & 0 \\
& 8 & 2 & 0 \\
& 7 & 2 & 1 \\
Média & 10 & 0 & 0 \\
\hline & 9 & 0 & 1 \\
& 10 & 0 & 0 \\
& 9,1 & 0,6 & 0,3 \\
\hline
\end{tabular}

" SCW - "snail conditioned water"

* Número de miracídios observados em cada uma das câmaras após 15 min da deposiçáo das larvas no canal $C$.
Tabela 6. Atração miraxodal exercida por água declorada colocada nos compartimentos $A, B$ e $C$, frente a miracidios de $S$. mansoni das geraçōes parental e $F_{16}$.

\begin{tabular}{lccc}
\hline \multirow{2}{*}{ Tipo de } & \multicolumn{3}{c}{ Número de miracídios* $^{*}$} \\
\cline { 2 - 4 } & Câmara A & Câmara B & Canal C \\
\hline Parental & 3 & 3 & 4 \\
& 4 & 3 & 3 \\
& 3 & 5 & 2 \\
& 2 & 3 & 5 \\
& 5 & 3 & 2 \\
& 5 & 3 & 2 \\
& 4 & 4 & 2 \\
Média & 2 & 5 & 3 \\
\hline F16 & 3 & 6 & 1 \\
& 6 & 2 & 2 \\
& 3,7 & 3,7 & 2,6 \\
\hline & 0 & 2 & 8 \\
& 1 & 2 & 7 \\
& 2 & 1 & 7 \\
& 1 & 0 & 9 \\
& 0 & 2 & 8 \\
& 2 & 2 & 6 \\
& 3 & 0 & 7 \\
Média & 1 & 3 & 6 \\
& 1 & 1 & 8 \\
& 3 & 1 & 6 \\
\hline
\end{tabular}

- Número de miracídios observados em cada uma das câmaras após $15 \mathrm{~min}$. da deposição de 10 larvas no canal $\mathrm{C}$.

geração selecionada. Entretanto os moluscos selecionados $\mathrm{e}$ suas $\mathrm{SCW}$ atraíram mais os miracídios, independentcmente se estas larvas eram ou não de gerações selecionadas (Tabelas 2 a 5).

Os miracídios sclecionados permaceram em grande númcro no canal do aparelho, quando se utilizou água declorada para o preenchimento das duas câmaras. Ao contrário, miracídios da geração parental dirigiram-se preferentemente para qualquer uma das câmaras (Tabcla 6).

\section{Análise Estatística}

Os resultados do experimento foram analisados através da lécnica de Gizzle e col. ${ }^{6}$ (1969), considerando-se como resposta o local onde se enconLrava o miracídio (câmara $\mathrm{A}$ ou $\mathrm{B}$, ou canal). $\mathrm{O}$ procedimento empregado estuda variações das probabilidades $\mathrm{Pa}, \mathrm{Pb}$ c $\mathrm{Pc}$ de miracídios dirigirem-se a $A, B$ ou $C$, respectivamente, em função dos fatores estudados.

O experimento permitiu a formação de populaçōes atravćs de todas as combinaçôes dos níveis dos fatores $\mathrm{cm}$ um total de oito.

A Tabela 7 mostra a importância de cada fator, ou combinação, nas distribuições de miracídidos pelos locais. O modelo ajustou bem os dados, deixando resíduo pequeno, com qui-quadrado 
Tabela 7. "Análise de Variância".

\begin{tabular}{lrrr}
\hline Fonte & GL & \multicolumn{1}{c}{$\begin{array}{c}\text { Qui- } \\
\text { quadrado* }\end{array}$} & Probabilidade \\
\hline Intercepto & 2 & 434,18 & 0,0000 \\
Tipo (Molusco, SCW) & 2 & 4,97 & 0,0834 \\
Molusco (Geração do & 2 & 28,74 & 0,0000 \\
Molusco) & 2 & & \\
Miracidio (Geração do & 2 & 0,74 & 0,6899 \\
Miracídio) & 2 & 7,12 & 0,0285 \\
Tipo/Molusco & 6 & 12,26 & 0,0564 \\
Resíduo & &
\end{tabular}

* Contribuição ao ajuste, segundo o modelo de Grizzle e col. ${ }^{6}$ (1969).

Tabela 8. Freqüência dos miracidios por local nos experimentos em que se utilizou molusco na câmara $A$.

\begin{tabular}{lccccc}
\hline & \multicolumn{4}{c}{ Geração do molusco } \\
\cline { 2 - 6 } & \multicolumn{3}{c}{$F_{16}$} & \multicolumn{3}{c}{ Parental } \\
& Freqüência & $\%$ & Freqüência & $\%$ \\
\hline Câmara A & 173 & 86,50 & 139 & 69,50 \\
Câmara B & 13 & 6,50 & 51 & 25,50 \\
Canal C & 14 & 7,00 & 10 & 5,00 \\
Total & 200 & 100 & 200 & 100 \\
\hline
\end{tabular}

Tabela 9. Freqüência dos miracídios por local nos experimentos em que se utilizou $\mathrm{SCW}^{*}$ na câmara $\mathrm{A}$.

\begin{tabular}{lrrcc}
\hline & \multicolumn{4}{c}{ Geração do molusco } \\
\cline { 2 - 6 } & \multicolumn{3}{c}{$F_{16}$} & \multicolumn{2}{c}{ Parental } \\
& Freqüência & $\%$ & Freqüência & $\%$ \\
\hline Câmara A & 171 & 85,50 & 143 & 71,50 \\
Câmara B & 24 & 12,00 & 47 & 23,50 \\
Canal C & 5 & 2,50 & 10 & 5,00 \\
Total & 200 & 100 & 200 & 100 \\
\hline
\end{tabular}

" SCW - "snail conditioned water".

Tabela 10. Freqüência dos miracidios por local, considerando a geração do miracídio, nos experimentos em que se utilizou molusco na câmara $A$.

\begin{tabular}{lrrccr}
\hline & \multicolumn{4}{c}{ Geração do molusco } \\
\cline { 2 - 6 } & \multicolumn{3}{c}{$F_{16}$} & \multicolumn{2}{c}{ Parental } \\
& Freqüência & $\%$ & Freqüência & $\%$ \\
\hline Câmara A & 159 & 79,50 & 153 & 76,50 \\
Câmara B & 34 & 17,00 & 30 & 15,00 \\
Canal C & 7 & 3,50 & 17 & 8,50 \\
\hline Total & 200 & 100 & 200 & 100 \\
\hline
\end{tabular}

Tabela 11. Freqüência dos miracídios por local, considerando a geração do miracidio nos experimentos em que se utilizou SCW* na cámara $A$.

\begin{tabular}{lrrrrr}
\hline & \multicolumn{4}{c}{ Geração do molusco } \\
\cline { 2 - 6 } & \multicolumn{3}{c}{$F_{16}$} & \multicolumn{3}{c}{ Parental } \\
& Freqüência & $\%$ & Frequência & $\%$ \\
\hline Câmara A & 161 & 80,50 & 153 & 76,50 \\
Câmara B & 30 & 15,00 & 41 & 20,50 \\
Canal C & 9 & 4,50 & 6 & 3,00 \\
\hline Total & 200 & 100 & 200 & 100 \\
\hline " SCW - "snail conditioned water".
\end{tabular}

12,26 (GL=6) e nível de significância $p=0,0564$. Vcrifica-se que a geração do molusco $\left(\mathrm{F}_{16}\right.$ e parental) foi significante $(\mathrm{p}=0,0000)$, mostrando que as probabilidades de atração são diferentes para as geraçōes $F_{16} c$ parental (ver Tabelas 8 e 9). Por outro lado, verifica-se que a atração não sofre cfeito da siluação do miracídio (Tabclas 10 e 11).

\section{Discussão e Conclusóes}

Brasio e col. ${ }^{1}$ (1985) já haviam verificado que excmplares de $B$. glabrata, pertencentes à população altamente susceptivel à infecção por S. mansoni, atracm, mais vigorosamente miracídios de população simpátrica do que moluscos $B$. tenagophila de populaçōes com baixo grau de susceptibilidade atraem miracídios também simpátricos.

Utilizando selcção genćlica por autofecundação das progênies susceplívcis, otleve-se em $\mathrm{F}_{16}$ uma população de $B$. tenagophila altamente susceptível (Tábcla 1). Através da utilização de $B$. tenagophila selecionada para o caráter susceptibillidade, confirmou-se o fato de que moluscos mais susceptíveis possuem maior poder de atração miraxonal. Verificou-se também que somente a população de planorbídcos interferiu na intensidade da atração. Os valores observados na atração miraxonal excrcida por moluscos da geração $F_{16}$ ou sua SCW, utilizando-se miracídios provenientes de $S$. mansoni mantidos $\mathrm{cm} B$. tenagophila sclecionada, mostraram-se maiores se comparados com os dados obtidos dos experimentos em que se utilizaram miracídios provenientes de $S$. mansoni mantidos $\mathrm{cm} B$. tenagophila não selccionada. Entrctanto, esta diferença não foi significativa. Conclui-se, portanto, que o fato de scr ou não miracídio proveniente de população de trematódco mantido $\mathrm{cm}$ moluscos sclecionados para o caráter susceptibilidade por várias gerações, não interfcriu no processo quimioláxico.

Os valorcs obscrvados na atração miraxonal, utilizando-sc SCW ou $B$, tenagophila scleciona- 
dos frente à miracídios selccionados ou não selecionados, foram muito semelhantes, fazendo crer que a seleção genética do molusco, quanto ao caráter susceptibilidade, não só facilita o desenvolvimento das larvas intra-molusco (Guaraldo e col. $.^{5}, 1981$ ) como atua na composição da SCW de maneira a tornar a atração miraxonal mais intensa.

ZANOTTI-MAGALHÃES, E. M. ct al. [Miraxonal attraction exercised by Biomphalaria tenagophila genetically selected for susceptibility]. Rev. Saúde públ., S. Paulo, 25: 134-8, 1991. The attraction of Schistosoma mansoni miracidea by snails genctically selected for susceptibility to infection is considered. Miracidea were exposed to snails or to snail conditioned water in a specially devised three-chamber glass apparatus. Ten miracidea were used for each test and were deposited in the middle chamber (a passage); after 15 minutes, them in each of the number of the three chambers were recorded. In one of the chambers, chosen randomly at each trial, either snails or SCW were used. The snails and the SCW were chosen from either selected snails (highly susceptible) or unselected. The miracidea were of two groups: they came either from susceptible snails in previous generation or were of unselected traits. The results show that only snail generation has importance for the attraction: selectd molluses of susceptible traits do attract the miracidea more.

Keywords: Biomphalaria, physiology. Schistosoma mansoni, physiology. Motor activity.

\section{Referências Bibliográficas}

1. BRASIO, B. C.; MAGALHÃES, L. A.; MILLER, J.; CARVALHO, J. F. Atração de miracídios de Schisto. soma mansoni por hospedeiros invertebrados: comportamento de miracídios frente a girinos de Hyla fuscovaria. Rev. Saúde públ., S. Paulo, 19: 18-27, 1985.

2. CIILRNIN, E. Bchavior response of miracidia of Schistosoma mansoni and other trematodes to substances emitted by snails. J. Parasit ., 56: 287-96. 1970.

3. CIICIRNIN, E. Penctrative activity of Schistosoma manso. ni miracidia stimulated by exposure to snail conditioned water. J. Parasit., 58: 209-12, 1972.

4. CIIERNIN, E. Some host-finding attributes of Schistosoma mansoni miracidia. Amer. J. trop. Med. Hyg., 23: 320-7, 1974

5. GUARALDO, A. M. A.; MAGALHÃES, L. A.; RANGEL H. A.; PAREJA, G. Evolução dos esporocistos de Schislosoma mansoni Sambon, 1907 em Biomphalaria glabrata (Say, 1818) e Biomphalaria tenagophila (D' Orbigny, 1835). Rev. Saúde públ., S. Paulo, 15: 436-48, 1981.

6. GRIZZLE, J. E.; STARMER, C. F.; KOCH, G. G. Analysis of categorical data by linear models. Biometrics, 25: 489-504, 1969.

7. KLOETZEL, K. Observaçōes sobre o tropismo do miracídio do Schistosoma mansoni pelo molusco Australorbis glabratus. Rev. bras. Biol., 18: 223-32, 1958.

8. ZANO'ITI-MAGALHÃES, E. M. Observaçöes sobre a capacidade de infeç̧ão do molusco vetor e a patogenidade do Schistosoma mansoni Sambon, 1907 no hospedeiro vencbrado, Campinas, 1987.[Tese de Doutoramento - Instituto de Biologia, Universidade Estadual de Campinas.]

Recebido para publicação em 12/10/1990 Aprovado para publicaçäo em 26/12/1990 